\title{
Inhalt
}

\section{Zur Einführung}

Albrecht Krieger, Dr. jur. h.c., Ministerialdirektor a. D. im Bundesministerium der Justiz, Bonn:

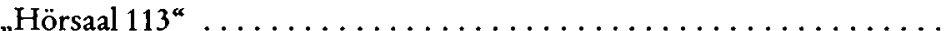

\section{Gewerblicher Rechtsschutz und Urheberrecht}

Friedrich-Karl Beier, Dr. jur., Dres. h.c., o. Professor an der Universität München, Geschäftsführender Direktor des Max-Planck-Instituts für ausländisches und internationales Patent-, Urheber- und Wettbewerbsrecht:

Mißbrauch einer beherrschenden Stellung durch Ausübung gewerblicher Schutzrechte? ...........................

Rainer Jacobs, Dr. jur., Rechtsanwalt, Honorarprofessor an der Universität zu Köln:

Photographie und künstlerisches Schaffen $\ldots \ldots \ldots \ldots \ldots \ldots \ldots$

Ulrich Krieger, Rechtsanwalt, Düsseldorf:

Zum Verhältnis von Monopolrecht und Vergütungsanspruch im Recht der Arbeitnehmer-Erfindungen . . . . . . . . . . . . . . .

Jon A.Lehman, Attorney at Law, New York, and Eric Stenshoel, Attorney at Law, New York:

Between Berne and Madrid: Movement of the United States Toward International Copyright and Trademark Protection ............

Wilhelm Nordemann, Dr. jur., Rechtsanwalt, Honorarprofessor an der Freien Universität Berlin:

Die „Tagebücher“ des Joseph Goebbels im Spannungsfeld von Besatzungs-, Persönlichkeits- und Urheberrecht . . . . . . . . . . .

Gerd Pfeiffer, Dr. jur., Präsident des Bundesgerichtshofes a. D., Honorarprofessor an der Fernuniversität Hagen:

Die geschäftliche Verleumdung nach $\$ 15$ UWG 
Gerhard Schricker, Dr. jur., Dres. h. c., o. Professor an der Universität München, Direktor des Max-Planck-Instituts für ausländisches und internationales Patent-, Urheber- und Wettbewerbsrecht:

Zur Geltung von Schiedsverträgen bei Anspruchsabtretung . . . . . . .

Oтro TePlitzky, Dr. jur., Richter am Bundesgerichtshof:

Die Warenzeichenveräußerung durch den Konkursverwalter im Konkurs über das Vermögen des Zeicheninhabers . . . . . . . . . . . .

Fritz Traub, Dr. jur., Honorarprofessor an der Universität Gießen, Vorsitzender Richter am Oberlandesgericht Frankfurt am Main:

Wettbewerbsrechtliche Nachahmungstatbestände und Klagebefugnis der

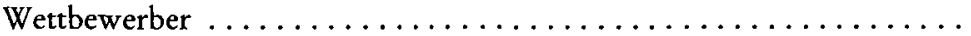

Ralf Vieregge, Dr. jur., Rechtsanwalt, Köln, und York StrothmanN, Dr. jur., Rechtsanwalt, Köln:

Preisausschreiben und Gewinnspiele als Werbemittel - zugleich Anmerkungen zur neueren Rechtsprechung $\ldots \ldots \ldots \ldots \ldots \ldots \ldots$

\section{Gesellschafts- und Konzernrecht}

Gerold Bezzenberger, Dr. jur., Rechtsanwalt und Notar, Berlin:

Zum Bezugsrecht stimmrechtsloser Vorzugsaktionäre .

Michael D. Blechman, Attorney at Law, New York:

Institutional Frameworks for Merger Control in the U.S. and the EC: The Social Advantages of "One Stop-Shopping" .............

Oliver C. Brändel, Dr. jur., Rechtsanwalt beim Bundesgerichtshof, Karlsruhe:

Mehrstimmrechtsaktien - ein in Vergessenheit geratenes Instrument der Beherrschung und des Minderheitenschutzes? . . . . . . . . . 175

HORST BRÄUtigam, Rechtsanwalt und Notar, Berlin:

Die Rechtsnachfolge in die Gesellschafterstellung als erledigendes Ereignis einer Ausschließungsklage? . . . . . . . . . . . . . . 189

Hans Erich Brandner, Dr. jur., Rechtsanwalt beim Bundesgerichtshof, Honorarprofessor an der Universität Karlsruhe:

Zur gerichtlichen Vertretung der Gesellschaft gegenüber ausgeschiedenen Vorstandsmitgliedern/Geschäftsführern . . . . . . . . . . . . 201

Wolfgang Gäbelein, Dr. jur., Rechtsanwalt, Düsseldorf:

Überlegungen zur Gestaltung eines europäischen Konzernrechts . . . . . 211 
Eckhart Gustavus, Dr. jur., Professor an der Fachhochschule für Verwaltung und Rechtspflege, Berlin:

Die Gesellschafterpublizität bei der $\mathrm{GmbH}$. . . . . . . . . . . . 22

Dieter Heckelmann, Dr. jur., o. Professor an der Freien Universität Berlin:

Die GbRmbH als (neue) Gesellschaftsform? . . . . . . . . . . . . . . 243

Ewald Hilger, Dr. jur., Rechtsanwalt am Oberlandesgericht, Düsseldorf: Zur Anwendbarkeit statutarischer Vinkulierungsklauseln bei der Übertragung von $\mathrm{GmbH}-\mathrm{Geschäftsanteilen} \mathrm{in} \mathrm{Ausführung} \mathrm{letztwilliger} \mathrm{Ver-}$ fügungen ..................... . . 259

Michael Hoffmann-Becking, Dr. jur., Rechtsanwalt, Düsseldorf:

Nachvertragliche Wettbewerbsverbote für Vorstandsmitglieder und Geschäftsführer . . . . . . . . . . . . . . . . . . . 273

Jürgen Lehmann, Dr. jur., Rechtsanwalt, Frankfurt am Main:

Die groben und die feinen Maschen des $\$ 126$ AktG . . . . . . . . . . 287

Marcus Lutter, Dr. jur., o. Professor an der Universität Bonn:

Die entschlußschwache Hauptversammlung . . . . . . . . . . . . . . 301

Herbert Messer, Dr. jur., Rechtsanwalt beim Bundesgerichtshof, Karlsruhe:

Die Kausalität von Mängeln des Verschmelzungsberichts als Voraussetzung für die Anfechtbarkeit des Verschmelzungsbeschlusses . . . . . . 321

Joachim Meyer-Landrut, Dr. jur., Rechtsanwalt, Düsseldorf:

Überschuldung als Konkursgrund . . . . . . . . . . . . . . . 335

Hans-Peter Müller, Rechtsanwalt, Vorstandsmitglied der Treuarbeit AG, Frankfurt am Main:

Bilanzrecht und Organverantwortung . . . . . . . . . . . 345

Welf MülleR, Dr. jur., Wirtschaftsprüfer, Rechtsanwalt, Steuerberater, Vorstandsmitglied der KPMG Deutsche Treuhand-Gesellschaft, Frankfurt am Main:

Die Änderung von Jahresabschlüssen - Möglichkeiten und Grenzen . . . 359

Hans-Joachim Priester, Dr. jur., Notar, Honorarprofessor an der Universität Hamburg:

Stille Reserven und offene Rücklagen bei Personengesellschaften Zur Bedeutung von $\$ 253$ Abs. 4 HGB . . . . . . . . . . . . 37 
Wolfgang Rosener, Dr. jur., Rechtsanwalt und Notar, Berlin:

Entwicklungen im Gesellschaftsrecht der DDR . . . . . . . . . . . . 397

Franz Jürgen Säcker, Dr. jur., Dr. rer. pol., o. Professor an der Universität Kiel:

Arbeitnehmerüberlassung im Konzern und Betriebsratsorganisation . . .

Johannes Semler, Dr. jur., Rechtsanwalt, Honorarprofessor an der Wirtschaftsuniversität Wien, Kronberg/Ts.

Sorgfaltspflicht und Verantwortlichkeit bei der Erstellung eines Gutachtens zur Vorbereitung eines Beteiligungserwerbs, insbesondere durch Wirtschaftsprüfer ....................

ECKART SüNNER, Dr. jur., Syndikus, Ludwigshafen:

Zur Abwehr feindlicher Unternehmensübernahmen in Deutschland . . . 457

Peter Ulmer, Dr. jur., o. Professor an der Universität Heidelberg:

Abfindungsklauseln in Personengesellschafts- und GmbH-Verträgen Plädoyer für die Ertragswertklausel . . . . . . . . . . . . . . . . . 477

Theodor Weigel, Rechtsanwalt und Notar, Frankfurt am Main:

Wirksamkeitserfordernisse für den Abschluß von Unternehmensverträgen zwischen Gesellschaften mit beschränkter Haftung . . . . . . . . .

Winfried Werner, Dr. jur., Rechtsanwalt, Frankfurt am Main, Honorarprofessor an der Universität Göttingen:

Die Ausgliederung. Ein Beitrag zum Entwurf eines Gesetzes zur Bereinigung des Umwandlungsrechts . . . . . . . . . . . . .

Harm Peter Westermann, Dr. jur., o. Professor an der Universität Tübingen, und Dietgard Klingberg, Dr. jur., Rechtsanwältin, Frankfurt am Main:

Vorkaufsrechte im Gesellschaftsrecht . . . . . . . . . . . . . 545

\section{Kartellrecht}

Oliver Axster, Rechtsanwalt, Düsseldorf:

Die Europäische Fusionskontrolle

Helmuth von Hahn, Dr. jur., Rechtsanwalt, Wiesbaden:

Der Beitrag der OECD zur Fortentwicklung und Harmonisierung der nationalen Kartellrechte und zur Bekämpfung von Wettbewerbsverzerrungen aus dem Bereich der öffentlichen Hand 
Wolfgang Kartte, Dr. jur., Honorarprofessor an der Universität Bonn, Präsident des Bundeskartellamts, Berlin:

Die gemeinschaftliche Nutzung von Warenzeichen im Spannungsfeld zwischen GWB und Warenzeichenrecht $\ldots \ldots \ldots \ldots \ldots \ldots \ldots$

Siegfried Klaue, Dr. jur., Direktor beim Bundeskartellamt, Honorarprofessor an der Freien Universität Berlin:

Räumliche Marktabgrenzung und Geltungsbereich des Kartellgesetzes . 625

Helmuth Lutz, Dr. jur., Rechtsanwalt, Stuttgart:

Die Marktmachtschwelle im deutschen Kartellgesetz bei zulässigen Kartellen und bei Zusammenschlüssen . . . . . . . . . . . . . . . .

Ernst Niederletthinger, Dr. jur., Ministerialdirektor im Bundesministerium der Justiz, Honorarprofessor an der Ruhr-Universität Bochum, Bonn:

Vier Prognosen zur europäischen Fusionskontrolle . . . . . . . . . 645

Herbert Sauter, Dr. jur., Direktor beim Bundeskartellamt, Berlin:

Ein Nachwort zur Europäischen Fusionskontrolle

Karsten Schmidt, Dr. jur., o. Professor an der Universität Hamburg:

EG-Gruppenfreistellung und nationale Gerichtsbarkeit

Claus Tessin, Dr. jur., Rechtsanwalt, Köln, und Andreas Röhling, Rechtsanwalt, Köln:

Die Beurteilung von Optionen nach deutschem und europäischem Kartellrecht ...........................

\section{Anwalts- und Standesrecht}

Gerhard Commichau, Dr. jur., Rechtsanwalt, Hamburg:

Zur Entstehung einer freien Advokatur in der DDR

Ottoarndt Glossner, Dr. jur., Docteur en droit, Rechtsanwalt und Notar, Kronberg/Ts.:

Das Standesrecht für den Schiedsrichter

Walter Kolvenbach, Dr. jur., Rechtsanwalt, Honorarprofessor an der Universität zu Köln, Düsseldorf:

Der Syndikusanwalt und das Anwaltsrecht

Günter SChardey, Dr. jur., Rechtsanwalt, Moers:

Neue Formen anwaltlicher Berufsausübung . . . . . . . . . . . 73

Joachim Schulze-Osterloh, Dr. jur., o. Professor an der Freien Universität Berlin:

Rechtsberatung durch Professoren der Rechtswissenschaft ........ 743 
